\title{
Análise retrospectiva dos fatores associados à distribuição da tuberculose bovina no estado do Rio de Janeiro
}

\author{
[Retrospective analysis of the factors associated to the distribution of bovine tuberculosis \\ in the State of Rio de Janeiro] \\ V.M. Oliveira ${ }^{1}$, A.H. Fonseca ${ }^{2}$, M.J.S. Pereira ${ }^{2}$, A.V. Carneiro ${ }^{1}$ \\ V.L.T. Jesus ${ }^{2}$, P.A.M. Alves ${ }^{3}$ \\ ${ }^{1}$ Embrapa Gado de Leite \\ Rua Eugênio do Nascimento, 610 \\ 36038-330 - Juiz de Fora, MG \\ ${ }^{2}$ Instituto de Veterinária da UFRRJ - Seropédica, RJ \\ ${ }^{3}$ PESAGRO - Seropédica, RJ.
}

\begin{abstract}
RESUMO
Fatores associados à ocorrência da tuberculose bovina (TB), em dados de 209 fazendas, foram avaliados por meio de análise de variância. Os dados utilizados foram provenientes do levantamento epidemiológico por demanda, realizado nos rebanhos do estado do Rio de Janeiro, no período de 1959 até 1989. As variáveis significativas na análise bivariada foram incluídas no modelo e analisadas conjuntamente pelo método do modelo linear generalizado. A taxa de prevalência da TB nos rebanhos foi de 49,8\%. As variáveis detectadas no estudo como as mais importantes para a ocorrência de TB foram: aleitamento $(\mathrm{P}=0,03)$ e as interações densidade $\mathrm{x}$ aleitamento $(\mathrm{P}=0,04)$ e produtividade $\mathrm{x}$ período $(\mathrm{P}=0,02)$. A importância da organização econômica da produção pecuária na ocorrência da TB pode ser evidenciada pelos resultados obtidos neste estudo. Este é um fator relevante em decorrência dos danos à saúde humana e animal.
\end{abstract}

Palavras-chave: bovino, tuberculose, epidemiologia, fatores de risco

\begin{abstract}
The factors associated to the occurrence of bovine tuberculosis (TB) in 209 farms were evaluated by variance analysis. Data came were colleted in an epidemiological investigation from herds in the State of Rio de Janeiro, examined from 1959 to 1989. The significant variables were analyzed and submitted to generalized linear model. The prevalence rate of TB in herds was 49.8\%. The most important variations detected in this study for occurrence of $T B$ were: sucking $(P=0.03)$ and interactions density vs. sucking system $(P=0.04)$ and productivity vs. sucking period $(P=0.02)$. The influence of the economical aspects of the farming systems on the occurrence of TB was confirmed by the results of this study. This is an important factor due to human and animal health hazards.
\end{abstract}

Keywords: bovine, tuberculosis, epidemiology, risk factors

\section{INTRODUÇÃO}

A tuberculose bovina (TB), doença contagiosa, causada pelo Mycobacterium bovis, é uma zoonose que tem como principal reservatório os bovinos, mas infecta uma grande variedade de espécies animais. A principal via de infecção para o homem é a digestiva, mas também ocorre pela via aerógena (Gil e Samartino, 2001). Para os bovinos, a via aerógena é a principal, sendo a via digestiva importante antes do desmame. Perdas por mortes de animais, redução da eficiência reprodutiva, queda na produção de leite e no ganho de peso, condenação de carcaças e restrição à exportação (Lilenbaum et al., 1998; Perez et al., 2002) caracterizam a importância econômica dessa enfermidade. 
Vários estudos utilizando técnicas multivariadas para análise dos fatores associados à ocorrência de tuberculose em rebanhos bovinos indicaram que as variáveis identificadas como sistema de produção, tamanho de rebanho, manejo, idade, raça, introdução de animais, presença de animais de corte e leite nos rebanhos agem como determinantes de prevalência diferenciadas de TB (Griffin et al., 1996; Marangon et al., 1998; Asseged et al., 2000; Omer et al., 2001; Perez et al., 2002). No Brasil, Belchior (2001) descreveu as seguintes variáveis: sistema de produção, grupo genético, sistema de ordenha, resfriamento do leite e monitoramento da produção como fatores de risco para a ocorrência de tuberculose.

O objetivo deste estudo foi avaliar os fatores associados à ocorrência da $\mathrm{TB}$, em rebanhos bovinos do estado do Rio de Janeiro, em amostra de demanda laboratorial, utilizando modelo linear generalizado binomial para distinguir a contribuição de cada variável, das interações e controlar o confundimento.

\section{MATERIAL E MÉTODOS}

Os dados, provenientes dos rebanhos de propriedades rurais do estado do Rio de Janeiro foram obtidos por meio de levantamento epidemiológico na base de dados de demanda laboratorial dos serviços do atual Laboratório de Reprodução Animal, convênio Embrapa/UFRRJ, no período de 1959 a 1989.

Segundo Jesus (2003), que trabalhou com amostra extraída do mesmo banco de dados, as informações foram obtidas de inquéritos sanitários que priorizavam as doenças da reprodução, em rebanhos cujos proprietários requisitavam os serviços para a implantação da inseminação artificial (IA). Os animais desses rebanhos foram submetidos aos exames de tuberculose, brucelose, leptospirose, campilobacteriose e tricomonose genital bovina. Nesse período, muitos proprietários, iniciantes na pecuária bovina, solicitavam exames por priorizar o controle sanitário do rebanho, e apenas alguns se preocupavam com a baixa fertilidade e a baixa produção. No período de 1970 a 1979 reduziram-se as solicitações para a implantação da IA, sendo o objetivo maior da procura o controle sanitário devido à baixa fertilidade dos rebanhos. No período de 1980 a 1989, houve maior preocupação com a baixa fertilidade, com o controle sanitário e, também, por haver suspeita da TB, os veterinários responsáveis procuravam auxílio dos órgãos competentes para diagnóstico e controle dessa enfermidade. Foram excluídas da análise as propriedades cujos dados apresentavam inconsistência para os propósitos deste estudo.

O teste de tuberculinização, segundo Langenegger et al. (1981), foi empregado para classificação dos rebanhos reagentes. Evidências de lesões na necropsia, assim como o isolamento do agente também foram utilizados, quando necessário. Nas análises para identificação dos fatores associados, consideraram-se as seguintes variáveis: assistência veterinária; aptidão do rebanho, categorizada em produção de leite com gado puro (LP), produção de leite com gado mestiço (LM) e sistema de produção duplo para leite e corte (LC); densidade animal $(\leq 0,50,>0,50 \leq 1$ ou $>1$ animal/ha); tamanho do rebanho $\quad(\leq 100,>100 \leq 200$ ou $>200$ cabeças $)$; produtividade $\quad(\leq 4,>4-\leq 7$ ou $>7$ litros/vaca/dia $)$; sistema de aleitamento (natural-N, artificial-A ou misto-M); critério para compra de animais, representado pela exigência de exames pelo produtor (não compra animais ou exige exames, às vezes exige, não exige); manejo de esterco (sim ou não); cuidados com recém-nascidos (total, parcial e sem cuidados) e mortalidade de bezerros $(\leq 5,>5 \leq 20$ ou $>20 \%)$ e tipo de demanda representada pelos períodos: 1959-1969, 1970-1979 e 1980-1989.

A associação entre a variável explicada (TB) e cada uma das variáveis consideradas explicativas foi testada pelo $\chi 2$, usando a correção de Yates, quando necessária, utilizando-se o procedimento tabelas do programa EpiInfo 2002 ${ }^{1}$.

A ocorrência de tuberculose foi submetida à análise de variância, por meio de modelo linear generalizado, assumindo a distribuição binomial dos dados. Para tanto, foi utilizado o procedimento MIXED disponível no pacote computacional $\mathrm{SAS} \AA$. As variáveis que ao teste de $\chi^{2}$ tiveram valores de $\mathrm{P} \leq 0,05$ e suas interações foram incluídas no modelo como efeitos fixos. $\mathrm{O}$ modelo geral utilizado na análise, em notação matricial, foi: $y=X \beta+e$, em que $y=$ vetor de observações; $X=$ matriz de incidência de efeitos fixos das variáveis classificatórias; $\beta=$ vetor de efeitos fixos e $e=$ vetor de efeito residual. O critério estatístico adotado na seleção das variáveis para a obtenção do melhor modelo foi o 
akaike's an information criterion (AIC), que tem como base o teste da razão de máxima verossimilhança, que consiste na comparação dos resíduos dos desvios do modelo com melhor ajuste aos demais.

\section{RESULTADOS E DISCUSSÃO}

A taxa de prevalência da TB nos rebanhos foi de 49,8\%. A alta percentagem de rebanhos infectados pode ser explicada, em parte, pelo tipo de amostra, isto é, de demanda. Lilenbaum et al. (1998) também observaram alta prevalência $(100 \%)$ em treze fazendas com histórico sugestivo de TB na região dos Lagos do estado do Rio de Janeiro. Em estudos com amostras aleatórias, normalmente as taxas são menores. Kantor e Ritaco (1994) relataram, porém, taxas variando de 6,2 a $26,3 \%$, obtidas dos resultados dos testes realizados em quatro regiões do Brasil, incluindo alguns rebanhos do estado do Rio de Janeiro, durante o ano de 1986.

Os resultados das análises de variância são apresentados na Tab. 1. Verificaram-se efeitos significativos $(\mathrm{P} \leq 0,05)$ das seguintes variáveis: aptidão do rebanho, densidade animal, produtividade, sistema de aleitamento e período.

O modelo que melhor explicou a ocorrência de TB nos rebanhos incluiu as variáveis: densidade, aptidão, produtividade, aleitamento e período e as interações densidade $\mathrm{x}$ produtividade, densidade $\mathrm{x}$ aleitamento $\mathrm{e}$ produtividade $\mathrm{x}$ período. Os resultados, resumidos, com os valores dos coeficientes, do erro-padrão e da probabilidade de cada variável e das interações, bem como o AIC do modelo, estão na Tab. 2.

À análise dos valores de $\mathrm{P}$ das variáveis e das interações, destaca-se que, apesar de a variável aptidão ter o valor de $\mathrm{P}=0,08$, a mesma foi mantida no modelo por melhorar a capacidade de entendimento do fenômeno como um todo, bem como a interação densidade animal $x$ produtividade, $\mathrm{P}=0,11$, o que foi evidenciado pelo AIC obtido com este modelo (AIC $=265,8)$.
As variáveis densidade $(\mathrm{P}=0,44)$ e período $(\mathrm{P}=$ 0,77 ) foram mantidas no modelo pela atuação nas interações.

Sistemas de produção que adotaram o aleitamento misto tiveram maior ocorrência de TB (78\% dos rebanhos positivos), que se assemelhou $(\mathrm{P}=0,09)$ àqueles com aleitamento artificial (58\% de rebanhos positivos) e se diferenciou $\quad(\mathrm{P}=0,0008) \quad$ daqueles com aleitamento natural $(43 \%$ dos rebanhos positivos). Este resultado está relacionado ao fato de que no aleitamento artificial o leite, proveniente de vacas infectadas, pode ser oferecido a crias de vacas sadias, aspecto salientado também por Morris et al. (1994).

A variável densidade, assim como aptidão, produtividade e aleitamento são importantes indicadores de tecnificação dos rebanhos, fruto das marcantes implementações tecnológicas no setor nesse período. Estes resultados estão de acordo com os achados de Belchior (2001), que apontou as variáveis: sistema de produção, grupo genético, sistema de ordenha, monitoramento da produção e resfriamento do leite, consideradas indicadores indiretos de tecnificação, como fatores de risco associados ao aumento de prevalência da tuberculose. Os resultados deste estudo reforçam, também, que as características da produção pecuária constituem fatores determinantes de ecossistemas diferenciados para doenças transmissíveis, o que foi comprovado por Obiaga et al. (1979) para a febre aftosa.

O desenvolvimento e a intensificação que ocorreram nos sistemas de produção pecuária, durante o período de estudo, podem ter contribuído para o aumento do aparecimento de novos casos de tuberculose bovina, principalmente em rebanhos leiteiros com animais de raças mais puras e, conseqüentemente, mais produtivas. Isso se deve, em parte, ao confinamento e ao maior contato entre os animais, aumentando a predisposição à infecção. 
Análise retrospectiva dos fatores...

Tabela 1. Análise bivariada dos fatores associados à tuberculose bovina de dados da demanda dos serviços do IPEACS/Embrapa, estado do Rio de Janeiro, 1959-1989

\begin{tabular}{|c|c|c|c|c|}
\hline Variável & $\mathrm{N}^{\circ}$ & Positivo & $\chi^{2}$ & $\mathrm{P}$ \\
\hline \multicolumn{5}{|l|}{ Assistência veterinária } \\
\hline Sim & 141 & 77 & \multirow{2}{*}{$3,50^{*}$} & \multirow{2}{*}{0,06} \\
\hline Não & 68 & 27 & & \\
\hline \multicolumn{5}{|l|}{ Aptidão do rebanho } \\
\hline LP & 57 & 36 & \multirow{3}{*}{6,10} & \multirow{3}{*}{0,05} \\
\hline LM & 96 & 45 & & \\
\hline $\mathrm{LC}$ & 56 & 23 & & \\
\hline \multicolumn{5}{|l|}{ Densidade (animal/ha) } \\
\hline$\leq 0,50$ & 81 & 36 & \multirow{3}{*}{6,64} & \multirow{3}{*}{0,04} \\
\hline$>0,5 \mathrm{a} \leq 1,0$ & 61 & 26 & & \\
\hline$>1,0$ & 67 & 42 & & \\
\hline \multicolumn{5}{|l|}{ Tamanho do rebanho ( $\mathrm{n}^{\circ}$ animais $)$} \\
\hline$\leq 100$ & 64 & 33 & \multirow{3}{*}{1,73} & \multirow{3}{*}{0,42} \\
\hline $101-200$ & 67 & 29 & & \\
\hline$\geq 201$ & 78 & 42 & & \\
\hline \multicolumn{5}{|l|}{ Produção (litros/propriedade/dia) } \\
\hline$\leq 150$ & 60 & 30 & \multirow{4}{*}{1,61} & \multirow{4}{*}{0,66} \\
\hline $151-450$ & 86 & 40 & & \\
\hline $451-600$ & 26 & 14 & & \\
\hline$>600$ & 34 & 20 & & \\
\hline \multicolumn{5}{|l|}{ Produtividade (litros/vaca/dia) } \\
\hline$\leq 4$ & 59 & 26 & \multirow{3}{*}{8,90} & \multirow{3}{*}{0,01} \\
\hline$>4 \mathrm{a} \leq 7$ & 79 & 34 & & \\
\hline$>7$ & 67 & 44 & & \\
\hline \multicolumn{5}{|l|}{ Sistema de aleitamento } \\
\hline Artificial & 38 & 22 & \multirow{3}{*}{12,36} & \multirow{3}{*}{0,002} \\
\hline Misto & 27 & 21 & & \\
\hline Natural & 143 & 61 & & \\
\hline \multicolumn{5}{|l|}{ Critério de compra } \\
\hline $\begin{array}{l}\text { Exige exames ou não compra } \\
\text { animais }\end{array}$ & 83 & 42 & \multirow{3}{*}{0,20} & \\
\hline Exige exames às vezes & 54 & 26 & & 0,90 \\
\hline Não exige exames & 65 & 34 & & \\
\hline Manejo de esterco & & & & \\
\hline Sim & 91 & 48 & & \\
\hline Não & 94 & 51 & 0,003 & 0,95 \\
\hline Cuidados recém-nascidos & & & & \\
\hline Total & 38 & 22 & & \\
\hline Parcial & 150 & 74 & 2,29 & 0,32 \\
\hline Não & 19 & 7 & & \\
\hline Mortalidade bezerros (\%) & & & & \\
\hline$\leq 5$ & 73 & 38 & & \\
\hline$>5 \mathrm{a} \leq 20$ & 100 & 49 & 0,98 & 0,61 \\
\hline$>20$ & 25 & 15 & & \\
\hline Períodos & & & & \\
\hline 1959-1969 & 104 & 39 & & \\
\hline $1970-1979$ & 61 & 36 & 12,93 & 0,002 \\
\hline $1980-1989$ & 44 & 29 & & \\
\hline
\end{tabular}

*Correção de Yates.

$\mathrm{LP}=$ produção de leite com gado puro; $\mathrm{LM}=$ produção de leite com gado mestiço; $\mathrm{LC}=$ sistema de produção duplo para leite e corte. 
Tabela 2. Análise de variância dos dados de demanda dos serviços do IPEACS/Embrapa, no estado do Rio de Janeiro, de 1959 a 1989, com o modelo de melhor AIC

\begin{tabular}{lcccc}
\multicolumn{1}{c}{ Variável } & Coeficiente & Erro-padrão & Valor z & P \\
\hline Densidade & 0,22128 & 0,28621 & 0,773 & 0,44 \\
Aptidão & $-0,35350$ & 0,20244 & $-1,746$ & 0,08 \\
Produtividade & $-0,58886$ & 0,36635 & $-1,607$ & 0,11 \\
Aleitamento & 0,77467 & 0,36358 & 2,131 & 0,03 \\
Período & 0,09301 & 0,31750 & 0,293 & 0,77 \\
Densidade x produtividade & 0,39911 & 0,25193 & 1,584 & 0,11 \\
Densidade x aleitamento & $-0,56441$ & 0,26831 & $-2,104$ & 0,04 \\
Produtividade x período & 0,59271 & 0,25704 & 2,306 & 0,02 \\
\hline
\end{tabular}

$\mathrm{AIC}=265,8$ (AIC: akaike's an information criterion)

Estudos em outros países, nos quais a análise de variância de múltiplas características foi empregada, dão suporte aos achados deste estudo. Embora a aptidão não tenha atingido nível de significância de $5 \%$, sua contribuição para a explicação do fenômeno tem sido apontada como importante, conforme observado por Marangon et al. (1998), que verificaram, em rebanhos da região de Veneto na Itália, que a presença de animais de corte e leite nas fazendas foi um fator de risco para a tuberculose. Resultados semelhantes foram obtidos por Omer et al. (2001), em Asmara, Eritea, que detectaram diferenças entre raças, uma vez que a aptidão leiteira no presente estudo esteve ligada ao padrão racial europeu. Os fatores ligados ao sistema de produção como produtividade, densidade e aleitamento podem explicar o fato de se atribuir maior risco à raça européia, contudo a resistência genética à infecção por $M$. bovis nunca foi conclusivamente comprovada (Morris et al., 1994).

\section{CONCLUSÕES}

O sistema de aleitamento e as interações densidade $\mathrm{x}$ aleitamento $\mathrm{e}$ produtividade $\mathrm{x}$ período são importantes fatores de risco para a ocorrência e distribuição da TB na população estudada. A importância da organização econômica da produção pecuária na ocorrência da tuberculose bovina foi evidenciada pelos resultados obtidos neste estudo.

\section{REFERÊNCIAS BIBLIOGRÁFICAS}

ASSEGED, B.; LÜBKE-BECKER, A.; LEMMA, E. et al. Bovine tuberculosis: a cross sectional and epidemiological study in and around Addis Abbaba. Bull. Anim. Health Prod. Afr., v.48, p.71-80, 2000.

BELCHIOR, A.P.C. Prevalência, distribuição regional e fatores de risco da tuberculose bovina em Minas Gerais, Brasil. 2001. 55f. Dissertação (Mestrado) - Escola de Veterinária, Universidade Federal de Minas Gerais, Belo Horizonte.

GIL, A.D.; SAMARTINO, L. Zoonosis en los sistemas de producción animal de las áreas urbanas y periurbanas de América Latina. Roma: FAO, 2001. p.16-22. (Livestock policy discussion. Paper, 2).

GRIFFIN, J.M.; MARTIN, S.W.; THORBUM, M.A et al. A case-control study on the association of selected risk factors with the occurrence of bovine tuberculosis in the Republic of Ireland. Prev. Vet. Med., v.27, p.217-229, 1996.

JESUS, V.L.T. Características da Tricomonose Genital Bovina em rebanhos no Estado do Rio de Janeiro: uma análise retrospectiva do período de 1958 a 2001. 2003. 96f. Tese (Doutorado) Instituto de Veterinária, Universidade Federal Rural do Rio de Janeiro, Seropédica, RJ.

KANTOR, I.N.; RITACO, V. Bovine tuberculosis in Latin América and the Caribbean: current status, control and eradication programs. Vet. Microbiol., v.40, p.5-14, 1994.

LANGENEGGER, J.; LANGENEGGER, C.H.; MOTA, P.M.P.C. Reações inespecíficas no diagnóstico alérgico da tuberculose bovina. Pesq. Vet. Bras., v.1, p.145-149, 1981.

LILENBAUM, W.; SCHETTINI, J.; RIBEIRO, E.R. et al. Tuberculose bovina: prevalência e estudo epidemiológico em treze propriedades de diferentes sistemas de produção na Região dos 
Lagos do Estado do Rio de Janeiro. Rev. Bras. Med. Vet., v.20, p.120-123, 1998.

MARANGON, S.; MATINI, M.; POZZA, M.D. et al. A case-control study on bovine tuberculosis in the Veneto Region (Italy). Prev. Vet. Med., v.34, p.87-95, 1998.

MORRIS, R.S.; PFEIFFER, D.U.; JACKSON, R. The epidemiology of Mycobacterium bovis infection. Vet. Microbiol., v.40, p.153-177, 1994.

OBIAGA, J.A.; ROSEMBERG, F.J.; ASTUDILLO, V.M. Las características de la producción pecuaria como determinantes de los ecosistemas de fiebre aftosa. Bol. Cent. Panam. Fiebre Aftosa, n.33-34, p.33-42, 1979.

OMER, M.K.; SKJERVE, E.; WOLDCHIWET, $Z$. et al. A cross-sectional study of bovine tuberculosis in dairy farms in Asmara, Eritrea. Trop. Anim. Health Prod., v.33, p.295-303, 2001.

PEREZ, A.M.; WARD, M.P.; TORRES, P. et al. Use of spatial statistics and monitoring data to identify clustering of bovine tuberculosis in Argentina. Prev. Vet. Med., v.56, p.63-74, 2002. 